\section{Visión Electrónica Más que un estado sólido \\ https://doi.org/10.14483/issn.2248-4728}

UNIVERSIDAD DISTRITAL

FRANCISCO JOSÉ DE CALDAS

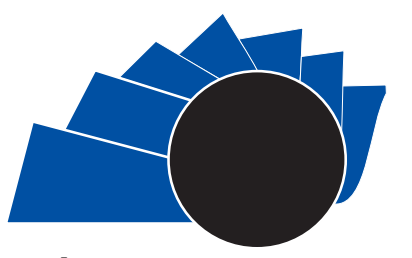

VISIÓN ELECTRONICA

Visión Investigadora

\title{
Impresora 3D open source desde el desarrollo de producto
}

\author{
3D printer open source from product development \\ Efraín Andrés Rodríguez Gasca ${ }^{1}$, Juan Pablo Rodríguez Gasca ${ }^{2}$, Luz Karime Hernández Gegen ${ }^{3}$
}

\section{INFORMACIÓN DEL ARTICULO}

Historia del articulo

Enviado: 02/02/2018

Recibido: 17/03/2018

Aceptado: 26/05/2018

\author{
Palabras clave: \\ Diseño de productos \\ Impresora $3 \mathrm{D}$ \\ Mudge \\ Reproducción de prototipos
}

\section{Keywords:}

Design of products

3D printer

Mudge

Reproduction of prototipes

\section{RESUMEN}

El presente artículo muestra el proceso de desarrollo de una impresora por aporte de hilo fundido basado en la metodología QFD (Quality Function Deployment). El diseño y la manufactura del producto, están descritos a través de las actividades de Desarrollo conceptual, Diseño a nivel de sistemas y Diseño detallado, donde cada una de ellas es evaluada y detallada. De igual manera, se tuvo en cuenta el diagrama de Mudge, con el fin de establecer los requerimientos para el diseño de la impresora y su grado de importancia. Como resultado del proceso, fue diseñada una máquina de impresión $3 \mathrm{~d}$ tipo RepRap, con una estructura mejorada para evitar desajustes y vibraciones, de igual manera se adicionaron piezas como una guía para el filamento y soportes para la electrónica y fuente de alimentación. La impresora cumplió con los requerimientos establecidos por los usuarios entre ellos su bajo costo y se encuentra operativa en la Universidad de Pamplona. Cabe destacar que la metodología empleada para el diseño, ha sido considerada como una ayudar en la toma de decisiones y selección de conceptos para la construcción.

\begin{abstract}
:
This article shows the process of developing a printer by contribution of cast thread based on the QFD methodology (Quality Function Deployment). The design and manufacture of the product are described through the activities of conceptual development, system-level design and detailed design, where each one of them is evaluated and detailed. In the same way, account was taken of the diagram of Mudge, in order to establish the requirements for the design of the printer and its degree of importance. As a result of the process, it was designed a 3D printing machine RepRap type, with enhanced structure to prevent misalignments and vibrations, Likewise pieces were added as a guide for the filament and brackets for electronics and power supply. The printer has complied with the requirements established by the users including its low cost and is operational at the University of Pamplona. It should be noted that the methodology used for the design, has been considered as an aid in decision-making and selection of concepts for the construction.
\end{abstract}

1 Ingeniero Mecatrónico, Universidad de Pamplona, Colombia. MSc. En Sistemas Mecatrónicos, Universidad de Brasilia, Brasil. Lugar de trabajo: Departamento de Ingeniería Mecánica y Mecatrónica, Universidad de Brasilia, Brasil. Correo electrónico: efrain.rodriguez@aluno.unb.br. ORCID: https://orcid.org/0000-0003-4926-2127.

2Ingeniero Electrónico, Universidad de Pamplona, Colombia. Lugar de trabajo: Departamento de Ingeniería Eléctrica, Electrónica, Telecomunicaciones y Sistemas, Universidad de Pamplona, Colombia. Correo electrónico: pablo.rodriguez@unipamplona.edu.co.orciD: https://orcid.org/0000-0002-4092-5898.

3 Ingeniera Mecánica, Universidad Francisco de Paula Santander, Colombia. Ph.D. En Tecnologías Avanzadas de Producción, Universidad de León, España. Lugar de trabajo: Grupo GIMUP: Universidad de Pamplona, Colombia. Correo electrónico: lukahege@,unipamplona.edu.co. orCID: https://orcid.org/0000-0001-5380-61 10.

Citar este artículo como: E. A. Rodríguez-Gasca, J. P. Rodríguez-Gasca y L. K. Hernández-Gegen, "Impresora 3D open source desde el desarrollo de producto", Visión electrónica, algo más que un estado sólido, vol. 1, no. 1, Edición especial, enero-junio 2018. DOI revista: https://doi.org/10.14483/issn.2248-4728. 


\section{Introducción}

En los últimos años, Impresión 3D, ha sido un término utilizado para describir un conjunto de tecnologías emergentes destinadas a la fabricación de partes mecánicas mediante la adición de material, en contraste con los procesos convencionales de manufactura substractiva. Desde sus inicios en los años 80s, estas tecnologías fueron creadas con la idea de manufacturar modelos prototipos de productos, directamente desde la data de un modelo virtual CAD-Computer Aided Design [1].

En la actualidad, la impresión 3D no sólo se limita al prototipado, sino que también permite la fabricación de partes mecánicas funcionales directamente para el usuario final. Consecuentemente, esto ha cambiado la forma en la que son fabricados muchos productos en la industria y ha propiciado una aceleración en el proceso de desarrollo de los mismos.

A lo largo de los años diferentes tipos de técnicas para la impresión 3D han surgido [2]. Sin embargo, la técnica del FDM-Funded Deposition Modeling se ha destacado como una de las más utilizadas para fabricación aditiva, debido principalmente a su facilidad de uso, economía y versatilidad. Esta técnica, aunque proporciona una baja calidad en el acabado del producto final, se ha tornado la alternativa más atractiva para investigadores, aficionados y pequeños emprendedores.

A pesar de que la impresión 3D es conocida desde hace más de 30, fue en la última década que se produjo un salto en los avances tecnológicos y en las aplicaciones relacionadas con este tipo de tecnología. La razón: el nacimiento del proyecto RepRap en el año 2005, creado por el profesor Adrian Bowyer en la Universidad de Bath, Reino Unido. Este proyecto, nace bajo la filosofía de desarrollar máquinas impresoras $3 \mathrm{D}$ de bajo costo con partes impresas por ellas mismas, es decir, con la capacidad de autor replicarse. De aquí que el nombre RepRap no es más que un acrónimo de Replicator Rápid Prototyping, una expresión utilizada para referirse a máquinas que son replicables a través de tecnologías de Prototipado Rápido, como también suele ser llamada la impresión 3D. Además de esto, el proyecto fue localizado en el ámbito del DIY-Do It Yourself y bajo el concepto de conocimiento abierto, o sea, que la información sobre cómo construir las máquinas, esté a la disponibilidad de todo el mundo de manera gratuita. Este proyecto se vio impulsado significativamente por el surgimiento, para la misma época, de del proyecto Arduino como tecnología de desarrollo libre, open source-open hardware.

De esta manera, el interés por el desarrollo de impresoras 3D ha sido una constante en los últimos años y una gran cantidad de diseños han sido propuestos. Entre las máquinas populares del proyecto RepRap se encuentran: Darwin, Prusa i2, Prusa i3, Printrbot, Rostock, Morgan, Wally, etc.

Con base en estos modelos, numerosos trabajos son encontrados hoy en la literatura [3,4]. La mayoría de ellos relacionados al diseño y la construcción. Sin embargo, en gran parte estos trabajos carecen de metodologías estructuradas que permitan la sistematización del proceso de desarrollo de estas máquinas, considerando la mayor parte de los aspectos envueltos en el ciclo de un producto: diseño, manufactura, embalaje, transporte, uso, mantenimiento, descarte.

En este contexto, el presente trabajo propone el desarrollo de una impresora 3D open source bajo el concepto de desarrollo de producto, utilizando una metodología estructurada basada en un modelo de referencia para el diseño y desarrollo de productos. La estructura se encuentra organizada de la siguiente manera: inicia con la introducción, en la sección 2 , es presentada la metodología de desarrollo la cual es dividida en la fase de desarrollo conceptual. La fase de diseño a nivel de sistema y diseño detallado. En la sección 3, se encuentran expuestos los principales resultados y en la 4 las conclusiones del trabajo.

\section{Metodología de Desarrollo}

El proceso de desarrollo de un producto posee una naturaleza compleja y multidisciplinar. Este proceso consiste básicamente en transformar un conjunto de entradas (materia prima, conocimiento, energía) en salidas deseadas (producto final) que buscan satisfacer necesidades específicas del cliente, utilizando una serie de operaciones, recursos, organizaciones e información. Sin embargo, la complejidad de este proceso diverge en relación al tipo de producto.

Al tratarse de un producto mecatrónico, como el caso de una impresora 3D, este es caracterizado por un alto grado de complejidad tecnológica, ya que su desarrollo demanda conocimiento sobre tecnologías mecánicas, electrónicas y computacionales. Siendo así, desarrollar un producto mecatrónico exige una sistematización a través de modelos de referencia para el desarrollo de productos, entendiendo modelo de referencia como el conjunto de directrices, procedimientos y actividades que auxilian la toma de decisiones en el proceso de desarrollo de un producto.

En este contexto, numerosos modelos han sido propuestos en la literatura, destacándose entre estos el modelo propuesto por [5] para el diseño y desarrollo de 
productos. Este modelo, representado en la [Figura 1], consiste en una secuencia de actividades que permiten concebir, diseñar, manufacturar y lanzar un producto al mercado, considerando así mismo su posible discontinuidad. El diseño y la manufactura del producto están descritos principalmente a través de las actividades de Desarrollo Conceptual, Diseño a Nivel de Sistema y Diseño Detallado. Al final de cada actividad son analizados y evaluados los resultados obtenidos a fin de validar los conceptos y criterios generados para el desarrollo del producto.

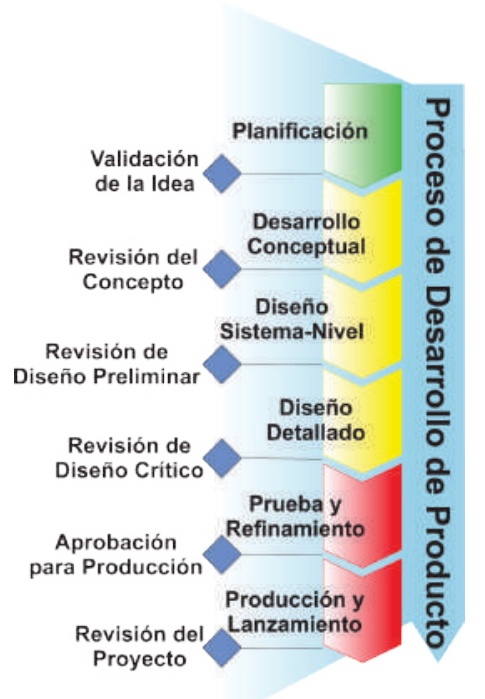

Figura 1. Modelo de referencia para el desarrollo un producto [5].

En este trabajo, el diseño y desarrollo de la impresora 3D open source ocurre a través de las fases de Desarrollo Conceptual, Diseño a Nivel de Sistema y Diseño Detallado, de acuerdo con el modelo de [5] . Estas fases son descritas a continuación:

\subsection{Desarrollo Conceptual}

En esta fase del proceso de desarrollo se busca definir un conjunto de especificaciones meta y atributos del producto. Es decir, las funcionalidades, características y nivel de tecnología que estarán presentes en el producto final, por lo cual es necesario conocer las expectativas y deseos del cliente. Siendo así, se inicia el proceso con el levantamiento de las necesidades del cliente y su posterior transformación en parámetros de ingeniería pasivos de prueba y medición, que finalmente se convertirán en especificaciones meta del producto (impresora 3D). Con la intención de facilitar esta tarea se ha empleado el método del QFD (Quality Function Deployment), el cual permite transformar sistemáticamente las necesidades del cliente en parámetros de ingeniería. Una descripción más amplia sobre el método QFD puede ser encontrada en [6,7].

Las necesidades del cliente fueron levantadas con base en una encuesta realizada a un grupo de personas con algún grado de conocimiento sobre impresión 3D y/o máquinas CNC. A continuación, las necesidades definidas en la voz del cliente fueron mapeadas y transformadas en requerimientos de usuario en un lenguaje más de ingeniería, teniendo como base en revisiones de literatura e los conocimientos del equipo de desarrollo en el área.

En total se establecieron 16 requerimientos de usuario para el diseño de la impresora $3 \mathrm{D}$, los cuales han sido agrupados en cuatro categorías principales: diseño, manufactura, uso y mantenimiento. Los requerimientos han sido listados en la tabla 1 y de igual forma fueron colocados en la matriz QFD. En el lenguaje del QFD los requerimientos de usuario suelen ser llamados "QUEs".

\begin{tabular}{|c|c|}
\hline Categorías & Requerimientos de usuario \\
\hline 1. Diseño & $\begin{array}{l}\text { 1.1-Estructura robusta; } 1.2-\text { Amplio espacio de trabajo; } \\
\text { 1.3-Bajo peso; } 1.4 \text { - Apariencia atractiva; } \\
\text { 1.5 Compacticidad }\end{array}$ \\
\hline 2. Manufactura & $\begin{array}{l}\text { 2.1-Fácil de montar; } 2.2 \text {-Bajo costo de fabricación e } \\
\text { montaje }\end{array}$ \\
\hline 3. Uso & $\begin{array}{l}\text { 3.1-Fácil de usar; } 3.2 \text {-Máquina rápida; } 3.3 \text {-Buena } \\
\text { calidad de impresión; } 3.4 \text {-Máquina segura; } 3.5 \text {-Largo } \\
\text { tiempo de operación; 3.6-Bajo costo de operación; 3.7- } \\
\text { Máquina silenciosa }\end{array}$ \\
\hline 4. Mantenimiento & $\begin{array}{ll}\text { 4.1-Fácil mantenimiento; } 4.2 & \begin{array}{l}\text {-Bajo costo de } \\
\text { mantenimiento }\end{array}\end{array}$ \\
\hline
\end{tabular}

Tabla 1 . Requerimientos de usuario agrupados en cuatro categorías. Fuente: elaboración propia

Los requerimientos de usuario son comparados par a par empleando un diagrama de Mudge, a fin de conocer la importancia y peso relativo de cada uno. En el diagrama de Mudge la importancia relativa de cada requerimiento es calculada como la sumatoria de la puntuación obtenida de una comparación par a par con el resto de requerimiento. Así mismo, el peso relativo de cada requerimiento se calcula dividiendo su importancia relativa entre la sumatoria de todas las importancias relativas de todos los requerimientos. Estas informaciones permiten priorizar en el diseño sobre los requerimientos más relevantes. Además, estas informaciones son requeridas como parámetros de entrada en la matriz QFD.

Como resultado del análisis con a través del diagrama de Mudge, se tiene que los requerimientos con mayor importancia para el diseño de la impresora 3D son: 1.1Calidad de impresión, 1.4-Amplo espacio de trabajo, 1.2Máquina rápida, 1.3-Longo tiempo de operación, 1.6- 
Impresión con varios tipos materiales e 4.1-Bajo costo de fabricación y montaje. De igual manera los requerimientos con menor importancia son: 3.3-Bajo peso y 3.6-Buena apariencia.

$\mathrm{El}$ siguiente paso fue desdoblar los requerimientos de usuario en parámetros de ingeniería que representen conceptos más físicos de la máquina y a su vez puedan ser mejor comprendidos por el equipo de desarrollo. De acuerdo con esto, se seleccionaron 16 parámetros de ingeniería que son listados en la [Tabla 2 y así mismo dispuestos en la matriz QFD. Los parámetros de ingeniería son llamados "COMOs" en el lenguaje del QFD.

\begin{tabular}{|l|l|}
\hline \multicolumn{1}{|c|}{ Categorías } & \multicolumn{1}{|c|}{ Requerimientos de ingeniería } \\
\hline 1. Diseño & $\begin{array}{l}\text { 1.1-Materiales resistentes; 1.2 -Volumen de trabajo ; } \\
\text { 1.3-Peso total de la máquina; 1.4-Acabado final; 1.5- } \\
\text { Dimensiones da máquina. }\end{array}$ \\
\hline 2. Manufactura & 2.1-Número de partes; 2.2-Precio total la máquina \\
\hline 3. Uso & $\begin{array}{l}\text { 3.1-Interfáz gráfica de usuario; 3.2 -Velocidad; 3.3 - } \\
\text { Presición; 3.4-Standards de seguridad de máquina; } \\
\text { 3.5-Tiempo de operación sin parar; 3.6-Consumo de } \\
\text { energía; 3.7-Nivel de ruido. }\end{array}$ \\
\hline 4. Mantenimiento & $\begin{array}{l}\text { 4.1-Partes de fácil a dquisición; 4.2 -Costo de } \\
\text { mantenimiento. }\end{array}$ \\
\hline
\end{tabular}

Tabla 2 . Parámetros de ingeniería seleccionados en relación a los requerimientos de usuario agrupados en cuatro categorías. Fuente: elaboración propia.

Una vez han sido definidos y colocados en la matriz QFD tanto los requerimientos de usuario como los parámetros de ingeniería, se procede a determinar el grado de relacionamiento entre estos. El grado de relacionamiento entre los requerimientos de usuario y los parámetros de ingeniería puede ser fuerte (valor de 9), medio (valor de 3), débil (valor de 1) o sin ningún tipo de relacionamiento (valor de 0 ). Al final para cada requerimiento de usuario son sumados los valores de relacionamiento con los parámetros de ingeniería, a fin de conocer cuál es el requerimiento que se ve más influenciado por los parámetros de ingeniería. En este caso el "Bajo costo de fabricación e montaje" fue el requerimiento de usuario con mayor influencia por parte de los parámetros de ingeniería, lo que quiere decir que las soluciones para la máquina deben ser basadas en materiales y procesos de manufactura de bajo costo.

Además de eso, la matriz QFD permitió obtener información acerca de la importancia y peso relativo de los parámetros de ingeniería con base en los relacionamientos con los requerimientos de usuario. Así mismo, fue establecida la correlación de cada parámetro de ingeniería par a par con los demás, buscando el equilibrio entre aquellos parámetros que se afectan negativamente, ya que el aumento de uno significa mayor afectación negativa sobre otro. Por ejemplo, al aumentar el volumen de trabajo de la máquina se afecta negativamente las dimensiones totales de la máquina, ya que estas aumentarían y lo que se busca es que la máquina sea compacta y su tamaño no sea relativamente exagerado.

Como resultado del análisis a través del método del QFD se obtienen las especificaciones meta de la máquina, que en otras palabras, serán las funcionalidades, características y atributos que el cliente final encontrará en el producto. Las especificaciones meta para la impresora 3D han sido listadas en la [Tabla 3] junto con su correspondiente parámetro de ingeniería y su respectiva tendencia deseada.

\begin{tabular}{|c|c|c|}
\hline Parámetros de ingeniería & Especificaciones meta & Tendencia \\
\hline Materiales resistentes & Aluminio, Polímeros, etc. & Target \\
\hline Volumen de trabajo & $200 \times 200 \times 300 \mathrm{~mm}$ & Aumentar \\
\hline Peso total de la máquina & Menos de $12 \mathrm{Kg}$ & Disminuir \\
\hline Acabado final & Proceso de pintura & Target \\
\hline Dimensiones da máquina & $400 \times 400 \times 400 \mathrm{~mm}$ & Disminuir \\
\hline Número de partes & Menos de 150 & Disminuir \\
\hline Precio total la máquina & Menos de COP 1.500 .000 & Disminuir \\
\hline Interfaz gráfica de usuario & Software fácil de usar & Target \\
\hline Velocidad & Máx. $250 \mathrm{~mm} / \mathrm{s}$ & Aumentar \\
\hline Precisión & $100 \mu \mathrm{m}$ & Aumentar \\
\hline $\begin{array}{l}\text { Estándares, de seguridad de } \\
\text { máquina }\end{array}$ & Standard ISO/TC199 & Target \\
\hline Tiempo de operación sin parar & En torno a 80 horas & Aumentar \\
\hline Consumo de energía & Menos de $200 \mathrm{~W} / \mathrm{h}$ & Disminuir \\
\hline Partes de fácil adquisición & Partes standard & Target \\
\hline Costo de mantenimiento & $\begin{array}{l}\text { Menos de } 3 \% \text { del valor total de la } \\
\text { máquina }\end{array}$ & Disminuir \\
\hline
\end{tabular}

Tabla 3. Set de especificaciones meta para la impresora 3D correspondientes a cada parámetro de ingeniería y su respectiva tendencia deseada. Fuente: elaboración propia

\subsection{Diseño Sistema-Nivel}

En esta fase, ocurre la formulación y selección de alternativas de solución que puedan atender las especificaciones técnicas definidas en la fase inmediatamente anterior. Se podría decir que es una transición de lo abstracto para lo concreto. El resultado de esta fase es la definición de la arquitectura global del producto-impresora 3D, descrita a través de sistemas, subsistemas y componentes.

Para generar las alternativas de solución son utilizados métodos de concepción basados en la creatividad del diseñador, los cuales pueden ser clasificados e intuitivos o sistemáticos. En este caso, se utilizó el método intuitivo del brainstorming para sugerir ideas que pudieran tornarse en principios de solución y el método sistemático de la matriz morfológica para seleccionar la mejor solución a un determinado problema, con base en una búsqueda sistemática dentro de una combinación de posibles alternativas de solución. 
Los principios de solución para la impresora 3D, que permitirán atender las especificaciones y meta establecidas, fueron formulados y seleccionados sistemáticamente a través de la matriz morfológica. Estos principios de solución permitieron definir la arquitectura de la máquina. En la [Figura 2] es mostrado un layout global de la arquitectura de la impresora 3D modelo prusa i2, señalando los principales subsistemas de la máquina. Como sistemas generales fueron consideradas las componentes mecánica, electrónica y computacional.

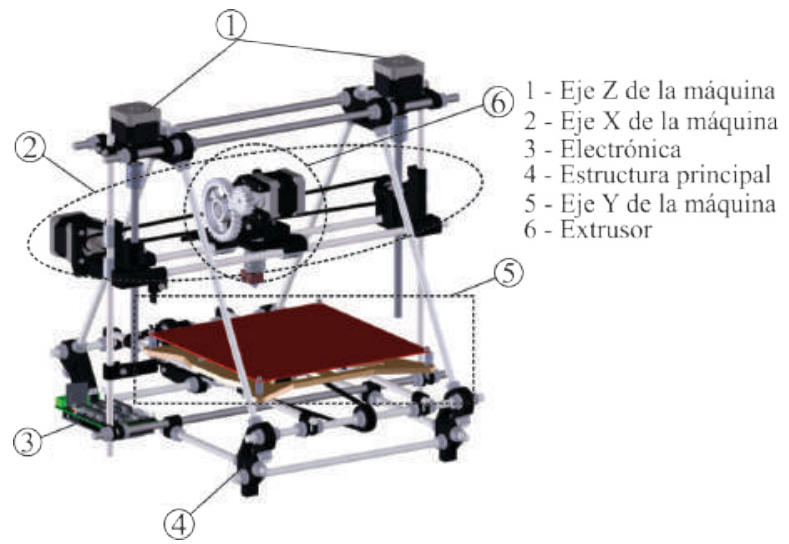

Figura 2 . Layout global de la máquina señalando los principales subsistemas. Fuente: elaboración propia

\subsection{Diseño Detallado}

En la fase de diseño detallado se busca definir una completa especificación de los materiales, geometrías, tolerancias, procesos de fabricación, partes fabricadas y comerciales, cálculos matemáticos, diagramas electroelectrónicos, etc., que hacen parte del plano de manufactura del producto.

En la [ Figura 3] son presentadas imágenes del proceso de construcción y montaje de la máquina. La mayoría de las piezas han sido manufacturadas en una impresora madre Makerbot z18 disponible en el laboratorio de Robótica de la Universidad de pamplona, en PLA negro a una altura de capa de $0.2 \mathrm{~mm}$. En total han sido 46 horas de impresión de para fabricar todas la partes de la máquina. Las partes que no son impresas, han sido adquiridas en el mercado local en Colombia. En total, el costo de los materiales para construcción de la máquina fue registrado en COP 1.250.000, sin contar el valor por hora de impresión para fabricar las piezas impresas.

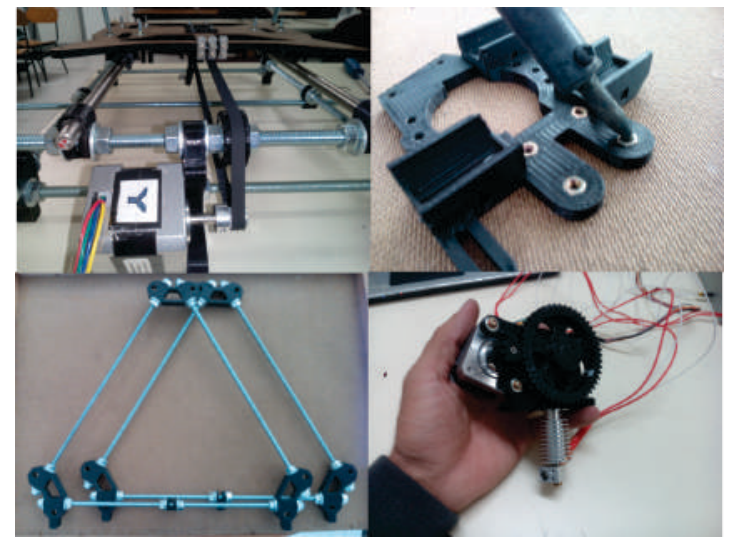

Figura 3 . Imágenes del proceso de construcción y montaje de la máquina. Fuente: elaboración propia

En la [Figura 4 ], es mostrado un diagrama de conexión de los componentes electro-electrónicos de la impresora 3D. Se utilizó una placa Arduino Mega 2560 como controlador con un shield de extensión tecnológica Ramps 1.4. Se puede apreciar el uso de 5 motores paso a paso NEMA 17 de 5,6 $\mathrm{Kg} \mathrm{cm}$, dos motores son destinados para el eje Z. Se hizo uso de una fuente de alimentación ATX de 12V. Tres finales de carrera para homing de recorrido mínimo, uno para cada eje de la máquina. El hotend del extrusor y la base térmica de impresión son conectados a su respectiva etapa de potencia en la Ramps 1.4. Los sensores de temperatura tanto para el hotend como para la base térmica han sido termistores de $100 \mathrm{k} \Omega$.

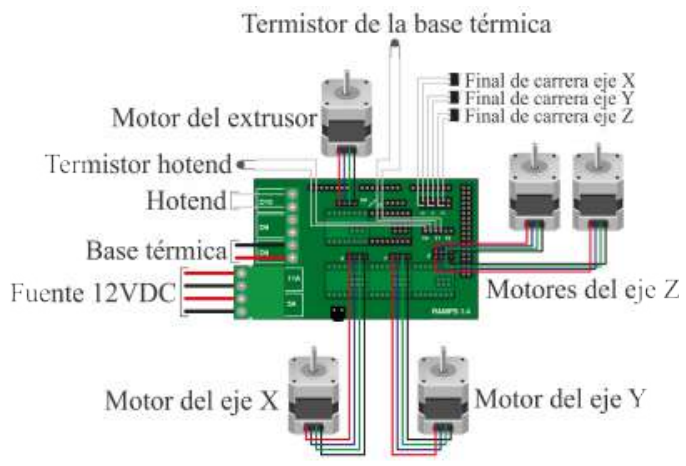

Figura 4 . Diagrama de conexión electro-electrónica de la máquina. Fuente: elaboración propia

\section{Resultados}

El resultado de este trabajo se ve reflejado en la máquina mostrada en la [Figura 5].

La impresora fue reforzada con algunas piezas en la estructura que no están presentes el diseño original del proyecto RepRap. Estas piezas cumple la función de 
evitar desajustes y vibración de la estructura principal. Además de eso se adicionaron las piezas para el cooler del extrusor, la guía para el filamento y los soportes para la electrónica y la fuente de alimentación. La distribución de la electrónica y la fuente de alimentación, fue mejorada y organizada respecto de la versión original de la máquina.

En la Figura 5 , la máquina desarrollada se aprecia imprimiendo partes para replicarse en una futura máquina clonada de sí misma. Estas piezas, fueron impresas a una velocidad de $160 \mathrm{~mm} / \mathrm{s}$ y una altura de capa de $0,2 \mathrm{~mm}$, obteniendo resultados satisfactorios del producto desarrollado.

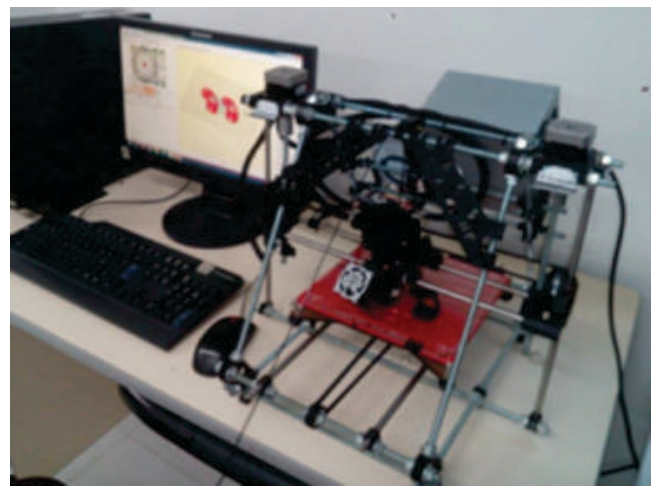

Figura 5 . Impresora 3D open source desarrollada. Fuente: elaboración propia.

\section{Conclusiones}

A pesar que las impresoras 3D de bajo costo aún no poseen un estado-capacidad comparativo con los mejores productos de este tipo existentes en el mercado, la relación costo-beneficio es un atractivo innegable, no solo para investigadores y aficionados, sino también para pequeños y medianos emprendedores.

Por otro lado, el diseño y la manufactura son actividades imprescindibles del proceso de desarrollo de un producto. El uso de un modelo de referencia para el desarrollo de productos permitió sistematizar gradualmente el diseño y desarrollo de la impresora 3D. Cabe destacar que las herramientas de diseño utilizadas, QFD y matriz morfológica, fueron importantes directrices de apoyo en la toma de decisiones para la generación y selección de conceptos y soluciones de ingeniería para el diseño de la impresora 3D.

Actualmente, la impresora 3D desarrollada es utilizada por estudiantes de Ingeniería Mecatrónica y afines de la Universidad de Pamplona para imprimir partes prototipos de sus proyectos académicos y de investigación.

\section{Referencias}

[1] S. Negi y R. K. Sharma, "Basics, Applications and Future of Additive Manufacturing Technologies: a Review", Journal of Manufacturing Technology Research, vol. 5, 2013, pp.75-96.

[2] L. Kumar, V. Kumar y A. Haleem, "Rapid Prototyping Technology for New Product Development", IJISET - International Journal of Innovative Science, Engineering \& Technology, vol. 3, no. 3, 2016, pp.287-292.

[3] E. Torres, J. León y E. Torres, "Diseño y construcción de una impresora 3D aplicando la técnica de prototipado rápido modelado por deposición fundida", Tercer Congreso Argentino de Ingeniería Mecánica III CAIM, 2012.

[4] E. Soriano, F. Blaya, M. Islán, S. Nuere y A. Rodriguez, "ETSIDI-Rap?: Impresora 3D portátil de código abierto", XXI Congreso nacional de ingeniería mecánica - At: ELCHE (SPAIN), 2016.

[5] K. Ulrich y S. Eppinger, "Product Design and Development", McGraw-Hill/Irwin, 2012.

[6] Y. Akao, "Quality Function Deployment: Integrating Customer Requirements into Product Design”, Taylor \& Francis, 2004.

[7] J. B. ReVelle, J. W. Morany C. A. Cox, "The QFD handbook", New York: Sons, John Wiley \& Sons, INC, 1998. 